\title{
Endothelin-1 and type III collagen alterations in the heart of rats following cisplatin-induced toxicity
}

\author{
QUAN-YONG HUANG ${ }^{1}$, XUE-FANG LI $^{2}$ and SHUI-PING LIU ${ }^{3}$ \\ ${ }^{1}$ Department of Pathology; ${ }^{2}$ School of Basic Medical Sciences, Dali University, Yunnan 671000; \\ ${ }^{3}$ Department of Forensic Pathology, Zhongshan School of Medicine, Sun Yat-Sen University, Guangzhou 510080, P.R. China
}

Received September 13, 2011; Accepted December 8, 2011

DOI: $10.3892 / \mathrm{mmr} .2011 .706$

\begin{abstract}
Cisplatin-induced heart damage is associated with enzymes and protein alterations. The purpose of this study was to investigate cisplatin-induced alterations in cardiac endothelin (ET)-1 and type III collagen following administration of cisplatin. Male Wistar rats were randomly divided into two groups: a control and a cisplatin group. The cisplatin group received cisplatin intraperitoneally (i.p.) at a single dose of $7 \mathrm{mg} / \mathrm{kg}$ on day 6 , while the controls were given the same amount of saline via the same route. On day 11, the rats were anesthetized and a thoracotomy was performed on all animals. Immunohistochemical analysis was performed to evaluate the protein expression of ET-1 and type III collagen. Sections were analyzed by digital image analysis. Results showed that the cardiac protein expression of ET-1 and type III collagen was altered following cisplatin-induced toxicity in rats. The expression of ET-1 and type III collagen was significantly increased after cisplatin treatment, supported by integrated optical density, when compared to the control group $(\mathrm{P}<0.05)$. The present findings indicate that such alterations may be reflected in abnormal cardiac function. Additionally, the proteins identified in this study may benefit investigations into the mechanisms underlying cisplatin-induced toxicity, thereby providing the necessary evidence for further research.
\end{abstract}

\section{Introduction}

Cisplatin is a chemotherapeutic agent widely used to treat a variety of solid tumors $(1,2)$. It is also an antineoplastic drug that causes an array of adverse effects on various organs and tissues (3). Nephrotoxicity is one of the major side-effects of cisplatin chemotherapy (4) limiting the promising efficacy of cisplatin (5). Cisplatin-based chemotherapy also has a variety of vascular side-effects (6). Acute administration of cisplatin

Correspondence to: Dr Quan-Yong Huang, Department of Pathology, School of Basic Medical Sciences, Dali University, Xiaguan Wanhua Road, Dali, Yunnan 671000, P.R. China

E-mail: hqy0726@126.com

Key words: endothelin-1, type III collagen, cisplatin-induced induces nausea/emesis and diarrhea (7). Chronic cisplatin may induce an enteric neuropathy characterized by changes in myenteric neurons associated with marked gastrointestinal motor dysfunction (7). Dysfunction in immune surveillance during anticancer chemotherapy of patients often causes weakness of the host defense system and a subsequent increase in microbial infections (8).

Cisplatin-induced toxicity is known to be involved in the changes of antioxidant enzymes (GST, SOD, CAT, GSH-Px and GSH), serum superoxide dismutase (SOD), glutathione (GSH), malondiethylaldehyde (MDA), nitric oxide (NO) (9) and DNA $(2,10)$. However, little is known about the changes in endothelin (ET)-1 and type III collagen expression in the heart following cisplatin-induced toxicity.

The present study used immunohistochemical analysis to evaluate the expression of ET-1 and type III collagen in the heart, and to determine whether or not the protein expression is altered with cisplatin-induced toxicity.

\section{Materials and methods}

Animals and study design. Sixteen healthy adult male Wistar rats, weighing 210-250 g, were used in this study. The animals were housed individually in stainless-steel wire-bottomed cages in an air-conditioned room at $25^{\circ} \mathrm{C}$ with $50 \%$ relative humidity and a 12-h light/dark cycle. Rats were fed with standard pellet chow and water throughout the experimental period. The procedures described in this study were approved by the Ethics Committee of the Sun Yat-Sen University.

The rats were randomly divided into two groups $(n=8$ per group). In the cisplatin group, Isotonic $\mathrm{Na}$ chloride was administered to animals by gavage for 10 days, and cisplatin was injected intraperitoneally (i.p.) to animals at a single dose of $7 \mathrm{mg} / \mathrm{kg}$ on day 6 . In the normal control group, the Isotonic Na chloride was administered to animals by gavage for 10 days and a single i.p. injection of isotonic saline was administered at the same dose on day 6 .

On day 11 , the animals were deeply anesthetized with sodium pentobarbital and a thoracotomy was performed. The hearts were then collected for light microscopy and immunohistochemical examination.

Histopathological examination. Heart specimens from each group were removed and examined histopathologically. The 


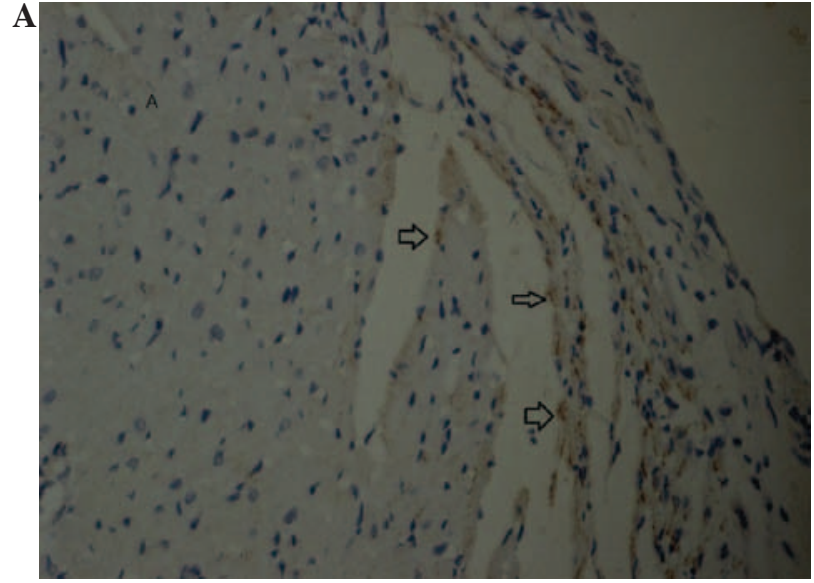

B

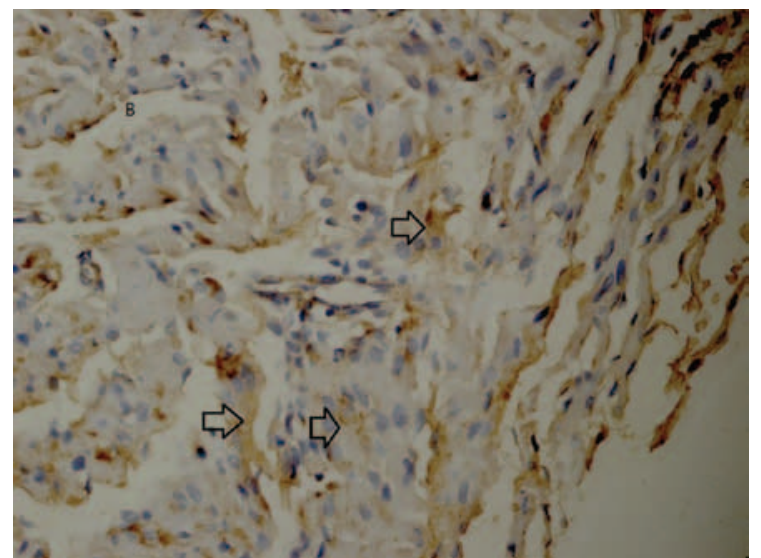

Figure 1. Effect of cisplatin exposure on ET-1 expression. Photomicrographs show a representative distribution of ET-1-positive expression in rat hearts from the (A) control and (B) cisplatin-exposed group. The photomicrographs were captured at a magnification of $\mathrm{x} 400$. Positive immunostaining appears as brown staining (arrow).

heart tissues were fixed in phosphate-buffered $4 \%$ formalin $(\mathrm{pH} 7.4)$ for $24 \mathrm{~h}$ and then embedded in paraffin. Sections were cut into $4-\mu \mathrm{m}$ slices and stained with H\&E. The slides were coded and semiquantitative analysis of the sections was performed in a blinded manner to the treatment by a pathologist. Pathological changes in these tissues were evaluated $(11,12)$.

Tissue sections and immunohistochemical staining. The rat hearts were immersed in $4 \%$ formaldehyde with phosphatebuffered saline (PBS; pH 7.2), prior to being embedded in paraffin, and sectioned coronally with a microtome into $4-\mu \mathrm{m}$ sections. Following deparaffinization, the sections were immersed in $0.3 \% \mathrm{H}_{2} \mathrm{O}_{2}$-PBS for 10 min and incubated with PBS containing $1 \%$ normal goat serum to reduce non-specific reactions. Following incubation with either rabbit anti-ET-1 or anti-type III collagen (Santa Cruz Biotechnology, Santa Cruz, CA, USA) overnight at $4^{\circ} \mathrm{C}$, tissue sections were washed three times in PBS and incubated with biotin-conjugated secondary antibody for $1 \mathrm{~h}$ at room temperature, according to the manufacturer's instructions. After washing, tissue sections were incubated for $10 \mathrm{~min}$ in streptavidin-peroxidase and then washed three more times in PBS. Positive signals were visualized with diaminobenzidine, followed by counterstaining with methyl green (13-15). PBS was substituted for primary antibody as the negative control.

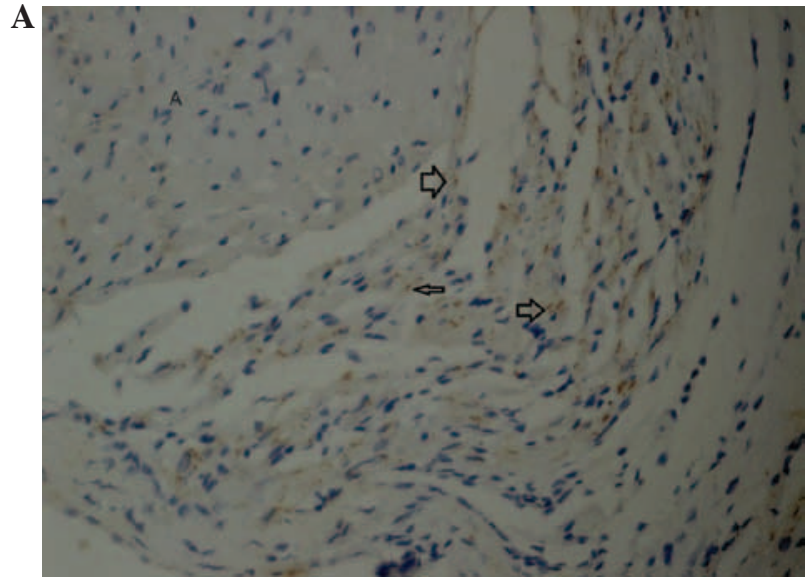

B

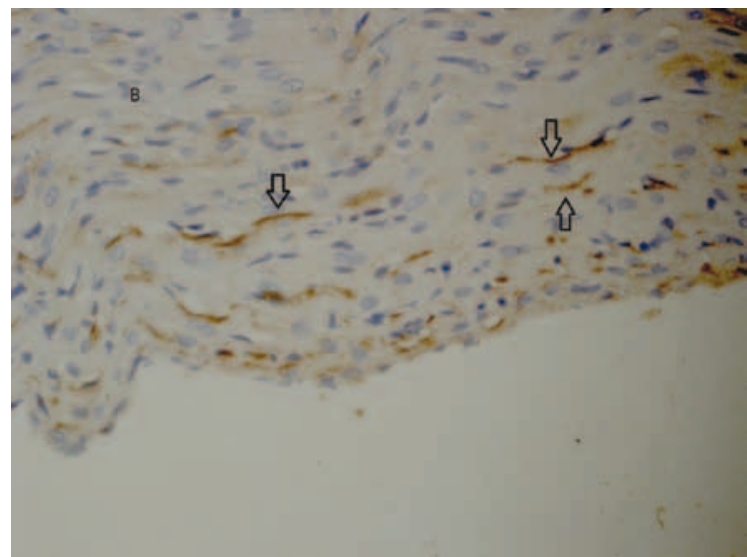

Figure 2. Effect of cisplatin exposure on type III collagen expression in rat hearts. Photomicrographs are a representative distribution of positive type III collagen expression in hearts from the (A) control and (B) cisplatin-exposed group. The photomicrographs were captured at a magnification of $\mathrm{x} 400$. Positive immunostaining appears as brown staining (arrow).

Table I. Integrated optical density (IOD) of ET-1 and type III collagen in the heart of rats.

\begin{tabular}{lcc}
\hline Group & ET-1 & Type III collagen \\
\hline Control & $0.0072 \pm 0.00043$ & $0.0067 \pm 0.00054$ \\
Cisplatin & $0.0159 \pm 0.00026$ & $0.0184 \pm 0.00071$
\end{tabular}

IOD per field is proportional to the total amount of staining. Total ET-1 IOD expression in rats subjected to cisplatin intoxication was significantly higher compared to the control hearts $(\mathrm{P}<0.05)$. Total type III collagen IOD expression in rats subjected to cisplatin intoxication was significantly higher compared to the control $(\mathrm{P}<0.05)$.

Total integrated optical density (IOD), a parameter representing the expression levels of ET-1 and type III collagen in cardiac tissue, was determined using a cast-grid microscope (MetaMorph/DP10/Bx41; UIC/Olympus, USA/JP) together with an image-analysis program (MetaMorph offline, version 4.65). Under a magnification of $x 400$, five images were captured in each immunostained section and the average was calculated $(14,15)$.

Statistical analysis. Statistical analysis was performed using SPSS 11.0 software. The results were presented as the 
means \pm SEM. The sources of variation were analyzed by the unpaired Student's t-test. $\mathrm{P}<0.05$ was indicative of statistical significance.

\section{Results}

Histological examination. Routine histological examination of rat hearts from each group revealed little morphologic change (data not shown).

Expression of ET-1 protein. Immunohistochemical staining was performed on formalin-fixed, paraffin-embedded sections from all parts of the heart, including the conduction system.

Positive staining for ET-1 antibody was detected mainly in the extracellular matrix. The ET-1 antibody was manifested as fine brown granularity. Two observers examined the sections independently.

The photomicrographs in Fig. 1A and B show the positive expression of ET-1 in the control (Fig. 1A) and the cisplatinexposed (Fig. 1B) rat hearts. Total IOD expression of ET-1 in rat hearts from each group is shown in Table I. Total IOD expression of ET-1 in hearts from rats following cisplatin intoxication was significantly higher than that in the control group (Table I, $\mathrm{P}<0.05$ ).

Expression of type III collagen protein. The type III collagen staining was detected mainly in the extracellular matrix. Positive stanining for type III collagen was manifested as fine brown granularity.

The distribution of type III collagen in the rat hearts of the control and cisplatin groups is displayed in Fig. 2A and B, respectively. Total type III collagen IOD in the heart of animals subjected to cisplatin intoxication was significantly higher than that of control hearts (Table I, P<0.05).

\section{Discussion}

Although chemotherapy with cisplatin is a widely used and effective cancer treatment, the unfavourable side-effects associated with it markedly decrease patient quality of life.

ET-1 is a potent vasoconstrictor that contributes to pathological processes responsible for cardiovascular disease (16). It plays a crucial role in the vascular function of most organ systems, both in physiological and pathophysiological conditions (17). In the pathological processes responsible for hypertension $(18,19)$, ET peptides and their receptors are involved in the physiological control of systemic blood pressure and body $\mathrm{Na}$ homeostasis (20).

ET-1 is known to induce cardiac hypertrophy through $\mathrm{G}$ protein-coupled receptors (21), and the activation of protein kinase $\mathrm{C}$ (PKC) has been implicated in alterations of myocyte function in cardiac hypertrophy and heart failure (22).

The multi-factorial regulation of ET-1 includes stimulated release by cytokines, macrophages and autonomic factors, and contributes to coronary plaque rupture and triggered cardiac events (23). ET-mediated coronary vasoconstriction is reduced with increasing exercise intensity, and decreased ET production during exercise likely contributes to the metabolic coronary vasodilation (24). Moreover, ET-1 contributes to the progression of cardiopulmonary pathology in rats
(25). Increased ET-1 in the heart, as revealed by our results, suggested that the tissues were greatly damaged when subjected to cisplatin-induced toxicity. Results of the present study indicated that cisplatin-induced toxicity increased ET-1 expression, which is associated with the impairment of heart function.

Type III collagen is a critical collagen that comprises extensible connective tissue, a crucial component of the extracellular matrix in the heart $(26,27)$, and appears to be involved in cardiac diseases. Myocardial fibrosis is the key factor that regulates left ventricular (LV) diastolic function, and collagen type III appears to be associated with cardiac fibrosis (28). It has been shown that cardiac fibrosis occurs mainly as a result of an increase of type III collagen (29).

The higher III/I ratio may contribute to progressive ventricular dilation and dysfunction in dilated cardiomyopathy patients (30). Collagen type I coexists with collagen type III in fibrils of both collagenous and reticular fibers (31). Collagen III synthesis is independent of the myofibroblast phenotype, and is reported to be regulated by differential growth factors and cytokines (32). Oxidative stress is reportedly associated with cisplatin-induced toxicity. The decrease of antioxidant enzymes (GST, SOD, CAT, GSH-Px and GSH) is involved in the cisplatin-induced toxicity process (9). The increased type III collagen in the heart, as shown by our results, suggests that the tissues were greatly damaged when subjected to cisplatin-induced toxicity. Findings of the present study have shown that cisplatin-induced toxicity caused an increase in type III collagen expression and this is related to the impairment of heart function.

The present results have shown that cardiac protein expression of ET-1 and type III collagen are altered following cisplatin-induced toxicity in rats. When exposed to cisplatininduced toxicity, the protein expression of ET-1 and type III collagen was significantly elevated. Thus, cisplatin-induced toxicity caused different alterations in heart proteins that may be correlated with the deterioration of cardiac function.

In conclusion, the present findings have shown that exposure to cisplatin-induced toxicity in rats leads to alterations of the heart proteins that may then be reflected in abnormal cardiac function. The proteins identified in this study may provide insight into the mechanisms underlying cisplatin-induced toxicity as well as the necessary evidence for further research.

\section{Acknowledgements}

This study was supported by a grant from the Natural Science Foundation of Guangdong Province, China (4203003), and the Guangdong Medical College (No. XQ0426).

\section{References}

1. Peterka M, Mandys V, Viale M, Cafaggi S, Peterkova R and Esposito M: Embryotoxicity of cisplatin and a cisplatin-procaine complex (DPR) studied in chick embryo. Neoplasma 49: 394-400, 2002.

2. Nijwening JH, Kuiken HJ and Beijersbergen RL: Screening for modulators of cisplatin sensitivity: unbiased screens reveal common themes. Cell Cycle 10: 380-386, 2011.

3. Fanzani A, Zanola A, Rovetta F, Rossi S and Aleo MF: Cisplatin triggers atrophy of skeletal C2C12 myotubes via impairment of Akt signalling pathway and subsequent increment activity of proteasome and autophagy systems. Toxicol Appl Pharmacol 250: 312-321, 2011. 
4. Chakraborty P, Roy SS, Sk UH and Bhattacharya S: Amelioration of cisplatin-induced nephrotoxicity in mice by oral administration of diphenylmethyl selenocyanate. Free Radic Res 45: 177-187, 2011.

5. Tripathi R, Samadder T, Gupta S, Surolia A and Shaha C: Anticancer activity of a combination of Cisplatin and fisetin in embryonal carcinoma cells and xenograft tumors. Mol Cancer Ther 10: 255-268, 2011.

6. Atalik KE, Keles B, Uyar Y, Dundar MA, Oz M and Esen HH Vasoprotection by melatonin and quercetin in rats treated with cisplatin. Indian J Exp Biol 48: 1188-1193, 2010.

7. Vera G, Castillo M, Cabezos PA, et al: Enteric neuropathy evoked by repeated cisplatin in the rat. Neurogastroenterol Motil 23: 370-378, 2011.

8. Oh GS, Kim HJ, Choi JH, et al: Activation of lipopolysaccharideTLR4 signaling accelerates the ototoxic potential of cisplatin in mice. J Immunol 186: 1140-1150, 2011.

9. Yousef MI, Saad AA and El-Shennawy LK: Protective effect of grape seed proanthocyanidin extract against oxidative stress induced by cisplatin in rats. Food Chem Toxicol 47: 1176-1183, 2009.

10. Cho EA, Oh JM, Kim SY, Kim Y and Juhnn YS: Heterotrimeric stimulatory GTP-binding proteins inhibit cisplatin-induced apoptosis by increasing XIAP expression in cervical cancer cells. Cancer Sci 102: 837-844, 2011.

11. Helin HO, Lundin ME, Laakso M, Lundin J, Helin HJ and Isola J: Virtual microscopy in prostate histopathology: simultaneous viewing of biopsies stained sequentially with hematoxylin and eosin, and alpha-methylacyl-coenzyme A racemase/p63 immunohistochemistry. J Urol 175: 495-499, 2006.

12. De Rossi A, Rocha LB and Rossi MA: Application of fluorescence microscopy on hematoxylin and eosin-stained sections of healthy and diseased teeth and supporting structures. J Oral Pathol Med 36: 377-381, 2007.

13. Smith PS, Parkinson IH and Leong AS: Principles of ploidy analysis by static cytometry. Clin Mol Pathol 49: M104-M111, 1996.

14. Dong J, Yin H, Liu W, Wang P, Jiang Y and Chen J: Congenita iodine deficiency and hypothyroidism impair LTP and decrease $\mathrm{C}$-fos and $\mathrm{C}$-jun expression in rat hippocampus. Neurotoxicology 26: 417-426, 2005.

15. Van Kuijk AW, Gerlag DM, Vos K, et al: A prospective, randomised, placebo-controlled study to identify biomarkers associated with active treatment in psoriatic arthritis: effects of adalimumab treatment on synovial tissue. Ann Rheum Dis 68 : 1303-1309, 2009.

16. Bagnato A, Loizidou M, Pflug B, Curwen J and Growcott J: Role of the endothelin axis and its antagonists in the treatment of cancer. Br J Pharmacol 163: 220-233, 2011.

17. Lima VV, Giachini FR, Hardy DM, Webb RC and Tostes RC: O-GlcNAcylation: a novel pathway contributing to the effects of endothelin in the vasculature. Am J Physiol Regul Integr Comp Physiol 300: R236-R250, 2011.

18. DeLoach S, Huan Y, Daskalakis C and Falkner B: Endothelin-1 response to glucose and insulin among African Americans. J Am Soc Hypertens 4: 227-235, 2010.
19. Liang F, Glascock CB, Schafer DL, et al: Darusentan is a potent inhibitor of endothelin signaling and function in both large and small arteries. Can J Physiol Pharmacol 88: 840-849, 2010.

20. Kohan DE, Rossi NF, Inscho EW and Pollock DM: Regulation of blood pressure and salt homeostasis by endothelin. Physiol Rev 91: 1-77, 2011

21. Hong HM, Song EJ, Oh E, Kabir MH, Lee C and Yoo YS: Endothelin-1- and isoproterenol-induced differential protein expression and signaling pathway in HL-1 cardiomyocytes. Proteomics 11: 283-297, 2011.

22. Puglisi JL, Yuan W, Timofeyev V, et al: Phorbol ester and endothelin-1 alter functional expression of $\mathrm{Na}+\mathrm{Ca} 2+$ exchange, $\mathrm{K}^{+}$, and $\mathrm{Ca}^{2+}$ currents in cultured neonatal rat myocytes. Am J Physiol Heart Circ Physio 300: H617-H626, 2011.

23. Burg MM, Soufer A, Lampert R, Collins D and Soufer R: Autonomic contribution to endothelin-1 increase during laboratory anger-recall stress in patients with coronary artery disease. Mol Med 17: 495-501, 2011.

24. De Beer VJ, Bender SB, Taverne YJ, et al: Exercise limits the production of endothelin in the coronary vasculature. Am J Physiol Heart Circ Physiol 300: H1950-H1959, 2011.

25. Lim KA, Kim KC, Cho MS, Lee BE, Kim HS and Hong YM: Gene expression of endothelin-1 and endothelin receptor a on monocrotaline-induced pulmonary hypertension in rats after bosentan treatment. Korean Circ J 40: 459-464, 2010.

26. Gramann M, Wendler O, Haeberle L and Schick B: Expression of collagen types I, II and III in juvenile angiofibromas. Cells Tissues Organs 189: 403-409, 2009.

27. Boudko SP, Engel J, Okuyama K, Mizuno K, Bachinger HP and Schumacher MA: Crystal structure of human type III collagen Gly991-Gly1032 cystine knot-containing peptide shows both $7 / 2$ and 10/3 triple helical symmetries. J Biol Chem 283: 32580-32589, 2008.

28. Terui G, Goto T, Katsuta M, Aoki I and Ito H: Effect of pioglitazone on left ventricular diastolic function and fibrosis of type III collagen in type 2 diabetic patients. J Cardiol 54: 52-58, 2009.

29. Tang M, Zhou F, Zhang W, et al: The role of thrombospondin1 -mediated TGF- $\beta 1$ on collagen type III synthesis induced by high glucose. Mol Cell Biochem 346: 49-56, 2011.

30. Soufen HN, Salemi VM, Aneas IM, et al: Collagen content, but not the ratios of collagen type III/I mRNAs, differs among hypertensive, alcoholic, and idiopathic dilated cardiomyopathy. Braz J Med Biol Res 41: 1098-1104, 2008.

31. Tateya T, Tateya I and Bless DM: Immuno-scanning electron microscopy of collagen types I and III in human vocal fold lamina propria. Ann Otol Rhinol Laryngol 116: 156-159, 2007.

32. Batra V, Musani AI, Hastie AT, et al: Bronchoalveolar lavage fluid concentrations of transforming growth factor (TGF)-beta1, TGF-beta2, interleukin (IL)-4 and IL-13 after segmental allergen challenge and their effects on alpha-smooth muscle actin and collagen III synthesis by primary human lung fibroblasts. Clin Exp Allergy 34: 437-444, 2004. 